\title{
ZUR ERINNERUNG POESIEALBEN AUS FLANDERN
}

\author{
Marcel VAN DEN BERG \\ B-2970 Schilde, Dianalaan 11. Belgien
}

Der Brauch, daß Kinder ein kleines Buch führen, in dem sie Zeichnungen und kurze Texte von Verwandten, Freunden und Bekannten sammeln, erfreut sich auch gegenwärtig großer Beliebtheit. Im Gegensatz zu anderen kurzlebigen Modeerscheinungen lebt diese Gepflogenheit bei Kindern in einem bestimmten Alter jedes Jahr wie eine Grippenepidemie wieder auf. Außenseiter dürften wenig Verständnis dafür haben, daß Wissenschaftler ein solches kindliches Phänomen ernst nehmen. Das Poesiealbum hat aber respektable Vorfahren, ist es doch aus dem „liber amicorum“ oder Stammbuch entstanden; weiter bieten vor allem die Alben der vorigen Generationen als „document humain“ oft interessante Einblicke in persönliche Erlebnisse (das Poesiealbum ist auch eine Art Ego-Dokument); und schließlich ist es eine wertvolle Quelle der Lebenskultur heutiger Kinder und Jugendlicher. Die Volkskunde hat zwar von Anfang an Interesse für bestimmte Aspekte der Kinderkultur wie Kinderlied und Kinderspiel gezeigt, aber erst seit den siebziger Jahren wurde die Kinderzeit als selbständige Lebensphase untersucht. ${ }^{1}$ Der Brauch des Poesiealbums ist mehr als ein Spiel oder eine „ästhetische Praxis“2 und hat eine gesellschaftliche Relevanz und eine soziale und sozialisierende Funktion.

Im Gegensatz zum „liber amicorum“ ist das Poesiealbum in Flandern kaum erforscht worden. In den Niederlanden erschien eine Sondernummer von Neerlands Volksleven $(15,1965$, S. 186-232) über das Poesiealbum, für unser Sprachgebiet die wichtigste Publikation über das Thema. Aber auch in diesem mehr als dreißig Jahre alten Artikel ist ein großer Teil des Textes dem „liber amicorum“ gewidmet (12 von 46 Seiten). In dem Katalog einer Ausstellung unter dem Titel Alba Amicorum. Vijf eeuwen vriendschap op papier gezet. Het album amicorum en het poëziealbum in de Nederlanden ('s-Gravenhage 1990) behandeln nur zwei Seiten das eigentliche Poesiealbum. Ab und zu stößt man auf einen kurzen Beitrag in nicht volkskundlichen und oft schwer zugänglichen Zeitschriften, u. a. in Damenblättern. In dem regiona-

${ }^{1}$ Siehe z. B. K. KöstLIN (Hrsg.): Kinderkultur. 25. Deutscher Volkskundekongreß in Bremen vom 7. bis 12. Oktober 1985. Bremen 1987; Spielwelten der Kinder an Rhein und Maas. Volkskundliche Unter-suchungen im Rheinland, 21, Köln 1993.

2 „Das Poesiealbum - eine ästhetische Praxis und mehr“ war der Titel eines Referates, gehalten von U. LANGBEIN auf der Österreichischen Volkskundetagung im Juni 1995 in Wien. Sie besorgte mir diesen Text und ich danke ihr für ihre Anregungen. 
len Fachblatt Kon. Havenwerktuigen S. K. lief 1995 eine Serie über Poesiealben aus Antwerpen. Darin gibt der Ingenieur A. HIMLER eine Sammlung von nach Motiv geordneten Eintragungen aus 11 Alben. Und - nochmals eine merkwürdige Koinzidenz: in der Novembernummer der niederländischen Zeitschrift Traditie las ich einen kurzen und offenbar gekürzten Artikel über das Thema. ${ }^{3}$ Im Nationalen Schulmuseum in Rotterdam lief 1996 eine attraktive, nach ästhetischen und thematischen Kriterien gestaltete Ausstellung. ${ }^{4}$ Die Organisatoren hatten nach einem Aufruf in den Medien nicht weniger als 1250 Exemplare bekommen, was beweist, daß das Album auch bei den erwachsenen Besitzern nicht ohne Bedeutung ist.

Vor allem in Deutschland wurde und wird das Thema untersucht. Ulrike LANGBEIN aus Berlin schrieb ihre Magisterarbeit darüber im Fach Europäische Ethnologie (1995).

Mit dem vorliegenden Beitrag ${ }^{5}$ will ich einerseits über den Stand meiner Untersuchungen berichten, zum anderen die Ergebnisse einer ersten Auswertung mitteilen und Erfahrungen und Anregungen weitergeben. Die Studie beschränkt sich auf Flandern und will vor allem den gegenwärtigen Brauch untersuchen.

Um das Thema und seinen Kontext zu studieren, sehe ich drei Zugänge: die Poesiealben selbst, die schriftliche Erhebung und das Gespräch mit Schulklassen und Kindern. Ich habe ungefähr 250 Bändchen durchgesehen, d. h. abgeschrieben oder fotokopiert. Das sind immerhin Tausende von Einträgen. Die meisten Bücher habe ich mir von Schülern und Studenten aus Grund-, Sekundar- und Hochschulen besorgen lassen. Das AMVC (Archiv und Museum für das Flämische Kulturleben) in Antwerpen besitzt ein Dutzend Alben aus dem Nachlaß flämischer Schriftsteller, Künstler und Intellektueller aus dem Ende des vorigen und dem Anfang dieses Jahrhunderts. Die Bibliotheken besitzen höchstens einige wertvolle Stammbücher aus vorigen Jahrhunderten. Um aber mehr über die Umstände des Brauches zu erfahren, z. B. wer (Junge oder Mädchen), wann, von wem und in welchem Alter ein Album bekommen hat, habe ich über verschiedene Kanäle Fragebögen mit je 36 Fragen verschickt. Es sind 1056 Exemplare eingegangen; die Frage nach dem Besitz wurde von 835 Respondenten positiv beantwortet. Ich war angenehm überrascht, als diese Umfrage auch viele Verse und Fotokopien von Albumblättern ergab, und sogar einige Briefe, in denen die Respondenten ausführlich berichteten, was das Poesiealbum für sie bedeutete. In dem oben erwähnten Referat hat LANGBEIN ein Plädoyer gehalten für eine Untersuchung in die Tiefe und eine subjektorientierte Forschungsstrategie. Nach ihrem Beispiel habe ich inzwischen auch eine Grundschule besucht.

3 P. F. Roggeveen: Rozen verwelken, schepen vergaan ... Poësieversjes: spiegel van de tijdsgeest. In: Traditie, 1 (3/1995), S. 20-23. Ich danke dem Autor dafür, daß er mir den ursprünglichen Text besorgt hat.

${ }^{4}$ Leider war bei der Ausstellung kein Katalog vorgesehen, aber das Museum besorgte mir ein Arbeitsdokument, wofür ich hier danke.

5 Es ist eine bearbeitete und aktualisierte Zusammenfassung des Artikels „Eeuwige vriendschap. Het poëziealbum in Vlaanderen“, in: Volkskultur an Rhein und Maas, 16 (1997), nr. 1-2, 45-68. 


\section{VOM „ALBUM AMICORUM“ ZUM SOGENANNTEN „KLASGENOOTJESBOEK“}

Das Poesiealbum ist aus dem „liber amicorum“ oder Stammbuch entstanden, das unter anderem Studenten ab dem 16. Jahrhundert auf ihren Reisen nach anderen europäischen Universitäten bei sich trugen und in dem sie Erinnerungen an Freunde, Professoren und andere wichtige Personen aufbewahrten, die sie kennengelernt hatten. Das Album war also ursprünglich eine ausgesprochen männliche Angelegenheit und eine Sache von jungen Erwachsenen, nicht von Kindern. Vor allem die Humanisten trugen zu der Verbreitung bei. Sogar in der ersten Hälfte des 19. Jahrhunderts gab es noch ab und zu Studenten, die diese Tradition fortsetzten, wie das Album von mr. Johannes Egberts Risseeuw (1798-1869) zeigt. Schon früh übernahm das gebildete Bürgertum diese Sitte und allmählich waren es immer mehr die jungen Mädchen aus diesen Kreisen, die ein Album führten. Ab 1870 ungefähr kann man bei uns von dem eigentlichen Poesiealbum sprechen; aus dieser Zeit stammt auch die neue Bezeichnung. Dann begannen nämlich die jungen Damen darin Gedichte mit einer bestimmten Thematik (Freundschaft, Liebe) zu sammeln. Die Texte waren oft sehr lang und meistens in französischer Sprache. Nach dem zweiten Weltkrieg wandelte sich abermals allmählich das Aussehen und der Inhalt des Albums; allerdings haben sich bestimmte Merkmale die Jahrhunderte hindurch gehalten. Die heutigen Besitzer sind sehr jung, können bisweilen kaum schreiben und demnach sind die Einträge oft sehr kurz und naiv und statt kalligrafischer Texte füllen farbige Kritzeleien die Blätter. Bei uns ist der Brauch fast allgemein unter Kindern in der Grundschule, auch bei männlichen Schülern, und bei Kindern aus allen Schichten der Bevölkerung.

Es gibt auch einige Innovationen. Neben dem traditionellen Poesiealbum mit weißen Blättern haben Verleger das vorgedruckte und illustrierte ,klasgenootjesboek“ oder „vriendjesboek“ (Mitschüler- oder Freundschaftsbuch) erfunden. Es ist ein Buch von 30 Seiten, in dem die Kinder bloß einige Daten ausfüllen müssen wie ihre Personalien, den Namen der Schule, ihre Hobbys, ihr Idol, ihre Wünsche, was sie später werden wollen, usw. Dieses „Steckbriefalbum“ läßt wenig Raum für Kreativität, aber hat, wie LANGBEIN meint, auch eine positive Seite. Schließlich geht es hier um Individualität und die Kinder sagen mehr über sich selbst als in den tradierten klischeehaften Reimen. Bei meinem Klassengespräch sagten einige Kinder spontan, daß sie das Freundschaftsbuch lieber hätten, weil man mehr über einander erfahre, aber die Mehrheit zog das klassische Album vor, weil darin mehr Platz zum Schreiben und Zeichnen sei.

Es gibt noch eine dritte, aber etwas weniger gebräuchliche Form. Manche Mädchen aus der Sekundarschule, Teenager und Backfische, führen ein ganz anderes „Freundschaftsbuch“, das sie auch „Textbuch“ nennen. Es ist ein liniiertes Schulheft, in das auf der einen Seite eine Illustration eingeklebt wird (eine Ansichtskarte mit einem Spruch), während auf der anderen Seite ein persönlicher Text mit intimen und sentimentalen Freundschaftsbeteuerungen und Gefühlsäußerungen steht. Auch Aphorismen, fremdsprachliche Zitaten und die traditionellen Albumverse treten 
auf. Das sogenannte Textbuch kann auch eine Sammlung von literarischen Texten sein, die der Besitzerin oder ihren Freundinnen besonders gefallen haben. Solche Textsammlungen gab es übrigens auch schon in der Vorkriegszeit.

\section{GESTALT UND AUFMACHUNG}

Die äußere Aufmachung variiert natürlich sehr, aber fast alle Alben haben das Hochformat. Einige sind viereckig und ein einziges Mal hat es eine ausgefallene Form (ein Pilz, ein Herz) oder folgt es dem Umriß der Zeichnung auf dem Einband (ein Hündchen). Im Durchschnitt ist es 13 bis $15 \mathrm{~cm}$ breit und 17 bis $20 \mathrm{~cm}$ hoch. Es hat 50 bis 80 Folios. Die Lederbände wurden allmählich durch Bände aus Kunstleder, Samt, Leinen oder Plastik ersetzt. Die älteren Exemplare sind einfach oder nicht verziert, tragen oft eine eingepreßte Aufschrift mit Goldprägung und Metallbeschläge. Die heutigen Alben sind sehr bunt. Die Aufzier kann abstrakt sein, aber meistens trägt die Einbandvorderseite eine Zeichnung, die die Kinder anspricht: spielende Kinder, Mädchen mit Blumen, Tiere, Figuren aus Disney-Filmen (The Lion King), usw. Andere Bändchen mit Fotos von jungen Leuten oder populären Idolen wenden sich an ältere Benutzer. Die Blätter sind unliniiert, eignen sich zum Zeichnen und können farbig sein. Einige Alben stecken in einer Pappschachtel oder haben ein Schloß.

Die Bezeichnung „poëzie“ oder „poëziealbum“ ist allgemein geworden nach 1850. In Flandern spricht man von einer ,poëzie“ (ein Kind schreibt z. B. ,pojizie“), in den Niederlanden sagen die Kinder ,poesie“ (Pussie). Die holländische Aussprache mag auf eine deutsche Herkunft hinweisen und wäre dann die niederländische Aussprache des deutschen Wortes. In Flandern ist der Name von der französischen Orthographie mit é: „poésie“ oder ,poésies“ abgeleitet.

\section{DIE BESITZER}

Nach 1870 und bis nach dem zweiten Weltkrieg war das Poesiealbum fast ausschließlich eine Sache für Mädchen. Das wird durch die Antworten auf die Frage, ob der Vater so ein Album besaß, bestätigt. Von 874 Informanten, die 1960 oder später geboren waren, haben nur 24 diese Frage mit ,ja“ beantwortet. Ein Mann aus dem Waasland (Jahrgang 1941) schreibt, daß in seinem Geburtsort das Album für Jungen nicht gebräuchlich gewesen sei. Der Brauch hat inzwischen so zugenommen, daß bei den Nachkriegsgenerationen fast alle Mädchen (97\%) daran teilnehmen, aber auch noch 56\% der Jungen. Zählt man nur die nach 1969 geborenen männlichen Respondenten, so bekommt man $75 \%$ positive Antworten. Dies hängt damit zusammen, daß die Besitzer immer jünger geworden sind. Die meisten Bändchen, die in Papierwarenhandlungen verkauft werden, wenden sich mit der Illustration auf dem Deckel an Mädchen oder allenfalls an kleine Kinder. Wenn man einem Jungen ein Album schenkt, sucht man sich weniger bunte Modelle aus. Doch finden noch immer viele 
männliche Gewährsleute das Führen eines Poesiealbums „blöd“ oder „lächerlich“ für Jungen. Natürlich treten sie wohl als Eintragende auf, so daß sie den Brauch mit aufrechterhalten. Vor einem „klasgenootjesboek“ scheinen sie sich weniger zu schämen.

Ich sagte schon, daß die Anfänger immer jünger werden. Mit 6 Jahren ist die untere Grenze wohl erreicht. 53\% der Kinder erhielten ihr Album als sie 6 oder 7 Jahre alt waren, $22 \%$ geben 8 oder 9 Jahre auf. Die meisten kriegten das erste Exemplar im ersten Schuljahr oder auf jeden Fall in der Grundschule, die bei uns 6 Jahre dauert. Viele fahren noch eine Zeitlang weiter, aber fast alle hören auf, wenn sie die Grundschule verlassen, also wenn sie 12 Jahre alt sind. Nur 22 Informanten haben nach ihrem 12. Lebensjahr noch ein Poesiealbum geführt. Das war früher anders; ältere Mädchenalben enden bisweilen erst mit der Verlobung oder der Heirat und eventuell folgen noch einige Kritzeleien des eigenen Kindes. Die heutige Beliebtheit der Gattung geht auch aus der Tatsache hervor, daß mehr als die Hälfte der Respondenten (57\%) mehr als ein Album gehabt hat. Trotzdem sind die Bändchen nicht immer voll. Die ersten Seiten werden schnell beschriftet, aber nachdem die nahen Verwandten und Freundinnen ihren Auftrag erfüllt haben, werden die Zeitintervalle größer.

Bei den älteren Generationen besaß nicht jeder ein Album. Es zirkulierte u. a. unter den Mädchen in Pensionaten, also unter Kindern aus gut bürgerlichen Kreisen. Aber auch Lehrer und Lehrerinnen und überhaupt Eltern aus anderen Berufsgruppen, die viel lasen, legten Wert auf das Poesiealbum.

\section{DIE SCHENKER}

Ein Poesiealbum kriegt man gewöhnlich geschenkt, obwohl ab und zu die Jugendlichen es auch selber kaufen. Ungefähr $47 \%$ der Besitzer hatten selber den Wunsch ausgesprochen, eines zu besitzen. Die Schenker sind meistens Frauen: die Mutter, die Großmutter oder eine Freundin. Der Anlaß ist natürlich derselbe wie bei anderen Geschenken: die Erstkommunion, der Geburtstag, der Nikolaustag, Weihnachten oder Neujahr.

\section{DIE EINTRAGENDEN}

Die Beiträge stammen von Freunden und Mitschülern, von Eltern, Großeltern, Geschwistern und anderen Verwandten, auch von Lehrern, Nachbarn und Bekannten der Familie. Die Umfrage zeigt, daß mehr als 70\% der Besitzer selber eine oder mehr Eintragungen machen. Sie schreiben dann z. B.: 78910 11, dit is van mezelf (sieben, acht, neun, zehn, elf; dies ist von mir selbst). Übrigens tauchen auch andere Namen oft mehrmals auf. Das Kind will natürlich aus seinem Album etwas Schmuckes machen und berücksichtigt bei der Wahl der Eintragenden ihr Talent. Darum werden auch oft Erwachsene gebeten. Bei Eltern mit Bekannten aus dem 
künstlerischen und kulturellen Leben bringt das originelle Texte und schöne Illustrationen. Vor allem Lehrer und Lehrerinnen sind gefragt. Andererseits will der junge Besitzer sein ausgeliehenes Album schon am folgenden Tag oder spätestens nach einer Woche zurückhaben, was dazu führt, daß manche Einträge übereilt gemacht werden.

\section{DIE QUELLEN}

Die Frage, woher man seine Texte nimmt, hat wenig genaue Angaben eingebracht. In ungefähr 10\% der Fragebögen werden Zeitschriften (Kinderblätter) oder Bücher erwähnt. Das können Ausgaben mit Albumpoesie sein oder Bücher mit Kindergedichten. Am meisten schreibt man Texte aus dem eigenen Album oder aus fremden Alben ab: ungefähr 34\% geben das zu. Eine Studentin besorgte mir ein Heft mit Albumreimen, die sie für späteren Gebrauch abgeschrieben hatte; eine Korrespondentin fotokopierte für mich das Heft, in dem sie Sprüche und Aphorismen gesammelt hatte. Auch wenn man die Bändchen von Kindern aus derselben Klasse vergleicht, sieht man die gegenseitige Abhängigkeit. Trotzdem antworten noch 30\% der Respondenten, daß sie, wenngleich nicht immer, selbst ihrer eigenen Phantasie und Inspiration folgen. Allerdings sind diese angeblich originellen Texte Variationen zu den üblichen Themen. Viel geplagte Lehrer oder Lehrerinnen schreiben und zeichnen überall dasselbe.

Was die Zeichnungen betrifft, sind die der Kleinsten deutlich eigene Arbeiten, wie man sie heute in jedem Schulzimmer antrifft. Sie dürften auch interessantes Studienmaterial für die Kinderpsychologie bieten. Ob die schöneren oder mehr akademisch ausgeführten Miniaturmalereien und -zeichnungen der älteren „Künstler“ Originelle oder Kopien sind, ist gewöhnlich nicht festzustellen. Die meisten Abbildungen sind wohl Nachzeichnungen und bisweilen sieht man noch die Spuren des Kohlepapiers. Kinderbücher, Ansichtskarten und vor allem Comic-Hefte und Zeichenfilme liefern das Bildmaterial. Bei älteren Kindern ist Imitation und Plagiat die Regel. Dies braucht nicht unbedingt negativ beurteilt zu werden. In dieser Lebensphase versuchen die Kinder, die Wirklichkeit naturgetreu wiederzugeben, sich in ihre Umwelt zu integrieren und verschiedene Identifikationsmodelle auszuprobieren.

\section{ILLUSTRATION UND DEKORATION}

Das Bändchen beginnt oft mit einem Eigentumstext, mit den Personalien oder einem eigenen Beitrag des Besitzers. Dieser reserviert mit Bleistift bisweilen die ersten Seiten für bestimmte Personen, von denen er einen Beitrag erwartet. Ab und zu wird systematisch ein Folio übersprungen, das gegebenenfalls später beschrieben wird. Die Zeichnung steht in Flandern jetzt meistens rechts, der Text links. Einträge ohne Zeichnung oder Zeichnungen ohne Inskription sind sehr selten, aber letztere 
kann äußerst kurz sein oder nur aus einer Identifikation bestehen. Nebst dem Namen ist auch das Datum ein wesentlicher Bestandteil der Eintragung. In älteren Exemplaren waren kalligraphisch geschriebene Texte die Hauptsache; Zeichnungen galten eher als Illustration und konnten sogar fehlen. Jetzt kommt es mehr auf die Zeichnung an und ist der Text oft nur eine Art Beischrift.

Auch die Seite mit dem Text wird fast immer ausgeschmückt, indem z. B. die Farbe, die Form und der Stil der Buchstaben oder Zeilen abgewechselt werden. Dann und wann werden Blümchen, Herzchen oder andere Figürchen über das Blatt gestreut. Man spielt mit der Anordnung der Wörter und Zeilen: Sätze laufen waagerecht, senkrecht oder diagonal über das Blatt, kreuzen einander, oder stehen auf dem Kopf.

Die Kinder haben offenbar ihre Freude an dem optischen Bild und an spielerischen, rebusartigen Formen. So wird das Datum bis zum Überdruß - und ab und zu auch ingeniös - versteckt. Sätze in griechischen Buchstaben oder in Spiegelschrift kommen auch einige Male vor. Die vier Silben VER GEET MIJ NIET (vergiß mich nicht) zieren die Ecken des Blattes. Umgefaltete Ecken oder Mini-Briefumschläge verbergen kurze Widmungen. Um die Jahrhundertwende produzierte man noch massenhaft Bögen mit gestanzten Bildern, die sogenannten Oblate, die Blumenkörbe, Girlanden, Kinder, Engelchen, Vögelchen usw. darstellten. Auch jetzt verwendet man moderne Aufkleber und allerhand ausgeschnittene Figuren. Wer wenig Zeichentalent hat, kann auch eine Karte einkleben. Auch Fotos, Andachtsbildchen (z. B. zur Kommunion) und sogar Speisekarten sollen an wichtige Momente erinnern. Bisweilen findet man einen Zettel, eine Trockenblume oder ein Kleeblättchen.

In den älteren Alben sind die Illustrationen mit Bleistift, Farbstift, Tusche oder Wasserfarbe ausgeführt. In den heutigen Exemplaren gebraucht man die gegenwärtigen Schreibmittel wie Kugelschreiber, Farbkreide und Filzstifte. Weil letztere durchdrucken, ist man dann gezwungen, das folgende Blatt leer zu lassen. Mißratene Zeichnungen werden ausgerissen oder mit dem nächsten Blatt verklebt.

Vor allem in älteren Bändchen sind Blumen wegen ihrer allegorischen Bedeutung das beliebteste Motiv. Diese Alben enthalten auch relativ mehr kunstvolle Bilder, u. a. aquarellierte Landschaften als die heutigen. Einige Eintragenden machen es sich leicht und machen eine skizzenhafte Bleistiftzeichnung mit der Unterschrift: „zum Färben“. Die Kinder, die heute spontan ihre Phantasie wirken lassen, stellen kleine Szenen aus ihrer Umwelt dar: das Kinderspiel, die Schule, den Garten, das Wohnzimmer, den Fernsehapparat. Sie zeichnen gern ein Kind, das den Auftraggeber darstellt. Nicht weniger beliebt sind Kaninchen, Bären, Eichhörnchen, Enten und andere Tiere in Menschenkleidung oder -haltung. Diese Abbildungen werden mit Märchenfiguren, Clowns, usw. abgewechselt. Aber vor allem der große Einfluß der Comic-Bücher und der Zeichenfilme fällt auf. Man läßt sich schließlich auch durch die Aktualität inspirieren und in der entsprechenden Jahreszeit schmücken Osterglocken, Nikolaus, Weihnachtsbäume und Neujahrswünsche die Blätter.

Selbstverständlich verraten die Zeichnungen auch das Interesse der Jungen: sie zeichnen andere Gegenstände als die Mädchen wie Fußballspieler, Flugzeuge, Rennwagen, Boote und Raumschiffe. 


\section{EINTRÄGE UND ALBUMPOESIE}

Bis zum zweiten Weltkrieg sind die Einträge in flämischen Alben oft in französischer Sprache, der Sprache der Bourgeoisie, wo das Album anfänglich hingehörte und auch die damalige Unterrichtssprache. Jetzt begegnen uns in Bänden älterer Jugendlichen $\mathrm{ab}$ und zu nicht nur französische, sondern auch deutsche oder englische Verse, Sprüche, Reime und Aphorismen. Der Einfluß der Schule und der Schriftsprache ist auch in der Wortwahl merkbar. Mundart wird selten gebraucht; die ,jij“-Formen sind den im alltäglichen Umgang üblichen „gij“-Formen überlegen.

Nur die Poesiealben der vorigen Generationen machen ihrem Namen Ehre und enthalten echte literarische oder originelle Gedichte. Beliebt waren u. a. V. Loveling, A. Nahon und G. Gezelle. In einigen Museumsstücken las ich Gelegenheitspoesie in der Handschrift von H. Conscience, P. Van Duyse, D. Sleeckx und anderen flämischen Autoren. Gedichte von bekannten Schriftstellern findet man heutzutage nur vereinzelt, und der Name Poesie trifft nur in dem Sinne zu, daß die gebundene Form noch immer der Prosa vorgezogen wird.

Man könnte eine Auslese, eine Art Anthologie der häufigsten und der originellsten Albumtexte machen. Die Thematik ist natürlich ziemlich beschränkt; die Norm läßt wenig Variationen zu. Die Inskriptionen können in ungefähr 20 Themenkreise eingeteilt werden. Allerdings können manche Inskriptionen in verschiedenen Gruppen untergebracht werden.

Auf dem ersten Blatt steht gewöhnlich eine Eigentumserklärung oder Präambel. Sie besteht einfach aus dem Namen und der Adresse, kann aber auch die Versform haben: Dit boekje is van mij zolang ik hoop te leven. Lenny is de naam in 't doopsel mij gegeven. Van Echelpoel is de naam van mijn vaderlijke stam. En Herentals de plaats waar ik ter wereld kwam. (Übersetzung: Dies Büchlein gehört mir solange ich werde leben. Lenny ist der Name in der Taufe mir gegeben. Van Echelpoel ist der Name meines väterlichen Stammes. Und Herentals der Ort wo ich zur Welt kam).

Gegenwärtig folgt darauf oft eine Art Reglement oder Gebrauchsanweisung für das Buch: Lees dit eerst: 1. Plak niets in deze poëzie. 2. geen ezelsoren. 3. geen bladzijden overlaten. 4. zeker NIET een prentje dichtplakken. 5. Zet steeds je naam en datum! 6. teken a. u. b. bij je naam (Lies zuerst folgendes: 1. Klebe nichts ins Album. 2. keine Eselsohren. 3. Seiten nicht überspringen. 4. Seiten nicht zukleben. 5. Immer Namen und Datum schreiben. 6. bitte eine Zeichnung machen).

Der heutige Besitzer fürchtet, daß sein Album nicht richtig behandelt werden könnte. Er will es rein halten und es auch zurückbekommen. Darum die Bitte, nicht mit Filzstiften zu schreiben oder die Warnung vor Diebstahl: Dit album is me lief. Wie het steelt is een dief. Die moet zitten op een latje. Met zeven spijkers in zijn gatje. Tot hij roept: genade heer. $O$ wat doet mijn gatje zeer. (Dieses Album ist mir lieb. Wer es stiehlt, ist ein Dieb. Der muß sitzen auf einem Brett. Mit sieben Nägeln in dem Hintern. Bis er ruft: Gnade, mein Herr. O was tut mir der Hintern weh!).

Was natürlich nicht fehlen kann, ist eine Identifikation des Eintragenden eventuell mit Widmung. In den aktuellen Bändchen steht oft ein kurzer Text, der nur aus dem eigenen Namen und dem des Auftraggebers besteht: Van ... voor ... (Von ... für ...) 
Het komt van ikke. Ik ben geen dikke. Wil je weten mijn naam. Hij zal hieronder staan. (Es kommt von ich. Ich bin nicht dick. Willst du wissen meinen Namen. Er wird hierunter stehen).

Es kommt nicht oft vor, daß der Besitzer mit einem Gruß bedacht wird. So steht unter einer Zeichnung, die ein Mädchen darstellt, das einen Brief in den Briefkasten wirft: Groeten uit Roosendaal (Grüße aus Roosendaal).

Die meisten Einträge sind einfache, spielerische Widmungen. Man schreibt z. B., daß man gerne den Wunsch des Auftraggebers erfüllt hat oder daß man zufrieden ist, daß die Zeichnung gelungen ist. 't Is op uw vraag. Dat ik het waag. ' $k$ Heb gedaan wat ik kon. Met potlood, verf en gom (Es ist auf deine Bitte, daß ich es wage. Ich habe mein Bestes getan mit Bleistift, Farbe und Radiergummi).

Da der Brauch in erster Linie die Freundschaft fördern soll, ist eine Freundschaftsbeteuerung das allgemeine Thema, das in allen möglichen Variationen vorkommt. Man besingt die Freundschaft und die Liebe und versichert dem Angesprochenen, daß man ihn nie vergessen werde. Op een bankje in het woud. Staat geschreven in het goud. Hoeveel ik van je houd. (Auf einem Bänkchen im Wald, steht geschrieben in Gold, wie sehr ich dich liebe). Twee roosjes op een stengel. Gij een schat en ik een engel. Gij een geel en ik een rood. Ik blijf je vriendin tot in de dood. (Zwei Röslein auf 'nem Stengel. Du ein Schatz und ich ein Engel. Du ein gelbes und ich ein rot(es). Ich bleibe deine Freundin bis in den Tod).

Aber auch der Eintragende selber will nicht vergessen werden und sieht ein, daß das Album ein Andenkenbuch ist, das später noch an Wert gewinnen wird. Der Aufruf zur Erinnerung ist ein Motiv, das noch stärker vertreten ist als das vorige: $I k$ dicht geen vers. Ik zing geen lied. Ik zeg alleen: Vergeet mij niet. (Ich dichte keinen Vers. Ich singe kein Lied. Ich sage nur: Vergiß mich nicht).

Mit der Bitte, die Freundschaft treu zu halten, ist oft ein Blick auf die Zukunft verbunden. Das Poesiealbum hat sowieso eine Zukunftsperspektive und manche Einträge weisen auf die Zeit hin, wenn das Kind erwachsen geworden oder gar alt geworden ist. Als je eens als grootmama. Netjes zit naast grootpapa. En je dan in dit boekje leest. Wil dan niet vergeten. Wie dit gedichtje heeft geschreven. (Wenn du später als Großmutter. Gemütlich sitzt neben Großvater. Und dann in diesem Album liest. Will dann nicht vergessen. Wer dieses Gedichtchen hat geschrieben.)

Um die Freundschaft zu unterhalten, schmeichelt man oft dem Auftraggeber mit einem Kompliment und lobt seine Tugenden, gegebenenfalls mit einiger Ironie: Kristineke is geen trineke maar een lief kineke. (Kristinchen ist ein liebes Kindchen).

Wünsche für eine schöne Zukunft, ein langes und glückliches Leben, usw. finden natürlich auch einen Platz im Poesiealbum: Kilometers vreugde. Hectoliters deugden. Maar geen gram verdriet. Dat is al wat ik u bied. (Kilometer Freude. Hektoliter Tugenden. Aber kein Gramm Verdruß. Das ist was ich dir wünsche).

Die älteren Alben stehen voll mit belehrenden, erbaulichen und frommen Aufforderungen, Ermahnungen, Geboten und Verboten. Man besang vor allem einen christlichen und einwandfreien Lebenswandel als Voraussetzung für ein glückliches Leben. Falscher Pathos kennzeichnet manche dieser Texte. In den heutigen Bänden sind die anmahnenden Imperative leichter, sogar spielerisch und freundlich und der 
Kindersprache angepaßt. Sie fordern das Kind auf, immer brav, keusch, fleißig, freundlich und munter zu bleiben und die Unschuld der Kinderjahre zu bewahren. Was ist die schönste Blüte. Auf dieser Blütenwelt?. Ein kindliches Gemüte. Ein Herz, das Gott gefällt (Winterslag). In grenznahen Gebieten, wie hier in Limburg, enthal-ten Poesiealben auch anderssprachige Texte.

Das Poesiealbum reflektiert die moralischen Auffassungen und Normen der Zeit. Vor allem in älteren Alben stehen viele Lebensweisheiten und Denksprüche, die eigentlich mit der Kinderheit nichts zu tun haben. Erwachsene schrieben früher sehr ernsthafte und freudlose Verse über das unabwendbare Schicksal, die Unvermeidlichkeit des Alterns, die schwere Bürde oder die Kürze des Lebens, über Krankheit und Tod. Kinder übernahmen ab und zu solche pessimistischen Texte, ohne sie voll zu erfassen. Jetzt haben die Lebensweisheiten einen leichteren Ton. Heranwachsende schreiben Sprüche und Aphorismen von Ansichtskarten ab, die u. a. von dem „Bond zonder Naam“ herausgebracht werden.

Vor allem die religiöse Botschaft ist aus den heutigen Alben verschwunden. Von der christlichen Religion inspirierte Texte sind wegen der fortschreitenden Entkirchlichung selten geworden. Meine Generation schrieb sie noch ab und zu unter dem Einfluß der Jugendbewegung oder der Schule: Uw boodschap dragen wij ,O Heer, door de wereld wijd. Getuigen zullen wij opdat allen U loven. (Ihre Botschaft tragen wir, Oh Herr, durch die weite Welt. Zeugen werden wir, damit alle Dich loben).

Ideologische Slogans, die durch die flämische Bewegung oder den flämischen Nationalismus inspiriert waren, kamen früher vereinzelt vor, unter anderem mit dem Symbol: $A V V-V V K$ (Alles voor Vlaanderen, Vlaanderen voor Kristus $=$ Alles für Flandern, Flandern für Christus). Ein Respondent erwähnt Kampflieder. Aus so einem Lied kommt auch folgender Text: Sta in de morgen. De lach op 't gelaat. Sterk voor uw volk. Fris voor uw taak. (Stehe in dem Morgen. Mit lachendem Gesicht. Stark für dein Volk. Zur Pflicht bereit). Im AMVC ist ein Album bewahrt, kein eigentliches Poesiealbum, mit Texten ausschließlich zum großniederländischen Gedanken. Die Jugendlichen von heute setzen sich vielmehr für Umweltschutz und für den Frieden ein.

Wie auch die Interviews gezeigt haben, schätzen die Kinder von heute vor allem lustige Texte und Zeichnungen. Humor und Nonsens, neckische, schalkhafte, absurde und etwas frivole Inskriptionen sind typisch für die heutige Generation. So finden sich in holländischen Alben Parodien bestimmter „evergreens“. Manche Reimchen haben gar nichts zu bedeuten oder wollen nur zum Lachen bringen. Sie sind aber nicht immer witzig und der Humor ist manchmal naiv oder infantil, und wenig originell. Alles in allem bleibt das Album auch sehr brav und derbe oder obszöne Texte findet man sozusagen nicht: Toen je nog een baby was. En je moeder je moest wiegen. Stak jij je beentjes in de lucht. En liet er eentje vliegen. (Als du noch ein Baby warst. Und dich die Mutter mußte wiegen. Stecktest du die Beinchen in die Höhe. Und ließest einen fliegen).

Weil es heutzutage mehr auf die Zeichnung ankommt und also erst entschieden wird, was man zeichnen will, liegt die Beschreibung des Bildes oder eine Anspielung nahe. Doch haben die meisten Zeichnungen gar nichts mit dem Text zu tun. Poesje 
groot, poesje klein. Altijd zal je mijn lieveling zijn. (Kätzchen groß, Kätzchen klein. Immer wirst du mein Liebling sein). Die Zeichnung kann natürlich auch sekundär sein und den Text illustrieren. Bei einer Zeichnung, die erst einen schlecht gelaunten und wenn umgekehrt, einen lachenden Mann darstellte, stand: Ben ik al eens wat slecht geluimd. Och kom dan draai ik maar mijzelve om. En eens mijn hoofdje anders om. Zeg ik: „Och, wat was ik dom“. (Und bin ich ab und zu mal schlecht gelaunt. Dann dreh ich mich einfach um. Und wenn mein Kopf steht andersum. Dann sag ich: Ach was war ich dumm).

Der Auftrag, etwas in ein Album zu schreiben und zu zeichnen ist eine Ehre, aber scheint auch eine Belastung oder sogar Belästigung zu sein. Einer der meist geäußerten Gedanken ist die Entschuldigung, daß man nicht besser reimen oder zeichnen könne. Zoals je wist. Ben ik geen artist. Maar toen ik eraan begon. Heb ik gedaan wat ik kon. (Wie du wohl wußtest. Bin ich kein Künstler. Aber als ich einmal daran begann. Habe ich getan, was ich konnte).

Neben den beiden Namen ist auch das Datum ein wesentlicher Bestandteil der Inskription. Wie oben schon erwähnt, wird es oft spielerisch versteckt.

Schließlich tragen Poesiealben bisweilen die Spuren persönlicher Erlebnisse oder öffentlicher Ereignisse und sind somit ein Zeitdokument. In der Kriegszeit wurden zum Beispiel Lebensmittelmarken eingeklebt. Kanadische Soldaten, die 1944 in einer Familie in Hoegaarden einquartiert waren, schrieben Worte des Dankes ins Album der Tochter. Ein 27jähriger Mann sammelte in einem selbst hergestellten Heftchen Zeichnungen von Mitgefangenen im Konzentrationslager in Beverlo.

\section{DIE BEDEUTUNG DES POESIEALBUMS}

Um die Bedeutung und Funktion des gegenwärtigen Poesiealbums zu erforschen, kann man nicht umhin, auch Schulklassen zu besuchen. Bei meinem Besuch in einer vierten Klasse der Grundschule hatte ich großen Erfolg: die Kinder hatten alle ihre Alben mitgebracht und waren sehr stolz auf ihren Besitz. Nachher hatte ich die Gelegenheit, an einige Kinder in einem persönlichen Gespräch Fragen zu stellen über die Rolle, die das Poesiealbum in ihrem Leben spielt, wie sie damit umgehen, was sie schön finden, usw. Man kann darüber hinaus auch Kinder von Bekannten befragen. Jane, ein Mädchen aus der sechsten Klasse zum Beispiel ist sehr kreativ und schrieb meistens ihre eigenen Texte; übrigens schreibt sie auch sonst ab und zu kleine Verse und führt ein Tagebuch. Sie ist sehr sozial und schließt niemanden aus. Was ihr Lehrer geraten hatte, das Album nur Kindern auszuhändigen, die schön zeichnen können, findet sie nicht gut. Hilary kriegte ihr Album, als sie in der ersten Klasse im zweiten Trimester war. Die beliebtesten Mädchen fingen damit an, und Hilary wollte auch mitmachen. Für sie hat das Album einen Erinnerungswert. So hat sie noch ein Andenken an eine Freundin, die inzwischen die Schule verlassen hat. Am liebsten hat sie Texte über sich selbst, die sagen, was die anderen von ihr halten. Whitney aus der vierten Klasse ist eine wirkliche Sammlerin: sie hat zwei Poesiealben und vier Steckbriefalben (Alle meine Schulfreunde) und sammelt auch Auf- 
kleber und Clowns. Steffi ist schlampig: sie hat ihr Steckbriefalbum verloren und kriegt fast keine Alben mehr von ihren Schulfreundinnen, weil sie sie zu lange liegen läßt. Übrigens mag sie viel lieber das klassische Poesiealbum, weil man darin sehr schön zeichnen könne. Sie hat ihr zweites Exemplar auf der Buchmesse gekauft und hat eine Vorliebe für das geschriebene Wort. Auch sie hat ein Tagebuch. Aus meinen Gesprächen mit Kindern zeigt sich, daß die Persönlichkeit, die privaten Lebensumstände, die Erziehung, usw. auch Einfluß haben auf die Bedeutung des Poesiealbums für das Kind und die Weise, wie es damit umgeht.

Auch in der Umfrage habe ich mich nach der subjektiven Einschätzung des Phänomens erkundigt und gefragt, welchen Wert das Album für die Respondenten habe und welchen Nutzen oder welche Funktion es habe. An erster Stelle will das Album natürlich Erinnerungen aufbewahren. Weniger zahlreich sind die Korrespondenten, die den Aspekt Freundschaft betonen oder auf die soziale Rolle hinweisen, die der Brauch in den Beziehungen mit anderen spielt. Wie andere Objekte aus der materiellen Volkskultur, muß das Poesiealbum in seinem Kontext betrachtet werden: aus Freundschaft geschenkt, wird es an Schulfreunde und Altersgenossen ausgeliehen, die also in den Freundeskreis aufgenommen oder in ihrer Freundschaft bestätigt werden. Kinder und Jugendliche sind vielleicht noch mehr als Erwachsene Modeerscheinungen und Trends unterworfen. Da sie in der Schule und in Vereinigungen dauernd in einer Gruppe leben, stehen sie unter starkem sozialem Druck: sie wollen sich benehmen wie die anderen. Das Poesiealbum ist ein periodisches Phäno-men, das jedes Jahr aufs neue eine ganze Klasse in Bewegung setzt, bis der Sturm der Begeisterung sich gelegt hat und die Liste der Kandidaten erschöpft ist, so daß man seine Freunde ein zweites Mal zur Last fallen muß. Das Führen eines Poesie-albums befriedigt auch die Sammelwut: Kinder haben ihre Freude daran, Zeichnun-gen und Reime auszutauschen.

Von 692 Personen, die ein Werturteil formuliert haben, sagen nur 31,4\% ausdrücklich, daß das Poesiealbum für sie einen großen Wert habe; $47,8 \%$ halten es für wenig und 21,4\% für nicht wertvoll. Trotzdem ist fast niemand bereit, es ohne weiteres abzugeben und einige wollen es sogar nicht ausleihen. Einige ältere Informanten legten spontan einen Brief bei, in dem sie ausführlich schrieben, wie lieb ihnen ihr Album sei. Für sie ist das Poesiealbum eine nostalgische Erinnerung an frühere Freunde und an die vergangene und sorglose Jugendzeit. Eine ältere Frau bewahrt die Textbücher ihrer Eltern wie Reliquien auf. Jungen mögen das Album weniger als Mädchen und finden es oft blöd oder mädchenhaft. Das mag mit den sentimenta-len und romantischen Freundschaftsäußerungen zu tun haben, die so typisch für Mädchen sind. Dennoch scheint sich der Brauch in den letzten Jahrzehnten auch bei ihnen stark verbreitet zu haben.

Das heutige Poesiealbum zeigt, wie Kinder miteinander umgehen, was sie interessiert, wie sie die Außenwelt in sich aufnehmen. Aber wegen der sozialen Kontrolle gibt es nur ein einseitiges Bild. Die Bedeutung der „erwachsenen“ Bücher ist wohl eine andere als die der Kinderalben. Die älteren Exemplare informieren uns z. B. über die Rezeption bestimmter Dichter und können unveröffentlichte Gelegenheitsgedichte enthalten. Auch in den jüngeren Bänden sind Erwachsene nicht unbeteiligt 
und somit ist das Album nicht nur ein Element der Kultur der Kinder, sondern auch der Kultur für Kinder. Es bringt zum Ausdruck, welche Erwartungen die Erwachsenen auf das Kind setzen. Es ist eine intime Reflektion gesellschaftlichen Lebens, ein Spiegel der Zeit und der Welt, in der es zustande gekommen ist. Das Genre ist alles andere als subversiv und der moralistische Ton überwiegt. Wohl wurden die Texte im Laufe der Jahre leichter, spielerischer und naiver. Inwiefern das Poesiealbum als Ratgeber auch Einfluß auf das Benehmen der Kinder hat, ist aber fraglich. Einige Erzieher in der ehemaligen DDR wollten dem Brauch entgegenarbeiten, andere sahen darin ein Instrument, mit dem sie die Jugend positiv beeinflussen könnten. Darin steckt natürlich eine große Gefahr für Indoktrination.

Ich sehe im Unterricht andere erzieherische Möglichkeiten für das Poesiealbum. In den Publikationen wird oft über die Verkitschung, Geschmack- und Gedankenlosigkeit der Eintragungen geklagt. Kinder sind große Imitatoren, sie äffen die anderen nach. Man sollte sie lehren, sich kreativ, eigenschöpferisch und natürlich zu äußern, sich selbst zu sein. Man sollte ihnen mehr Gefühl für Schönheit und echte Poesie beibringen und ihre verbalen Ausdrucksmöglichkeiten entwicklen, die sie ja haben, wie z. B. das Kinderlied zeigt. Dann gäbe es vielleicht weniger Banalitäten und Klischees in den Kinderalben. Das ist genau, was ein Volksschullehrer aus Gent mit seinen Schülern versucht. Er hat selbst mit 10 Jahren von seinen Eltern (Vater Metallarbeiter) ein Poesiealbum bekommen und bis zum 18. Lebensjahr geführt. Er erzählt seinen Schülern, daß ursprünglich nicht die Zeichnung, sondern die Poesie die Hauptsache gewesen sei und wie schön und gepflegt die früheren Alben gewesen seien. Er macht mit ihnen Gedichte und sagt ihnen, daß sie auch fürs Album ihr eigenes Gedicht machen sollten. Eine Frau, für die Poesie und Bücher immer viel bedeutet haben, schrieb mir in einem sehr langen Brief, daß sie ihren Enkelkindern rate, etwas Persönliches zu erdichten und das Herz sprechen zu lassen. Diese zwei Beispiele zeigen, daß das Poesiealbum eine Rolle spielen kann und spielt in dem Umgang mit der Ästhetik. Inzwischen bleibt es auch in seinen heutigen Erscheinungsformen eine wertvolle Quelle der Lebenswelt und Kultur von Kindern und Heranwachsenden. 